\title{
On PT Symmetry Systems: Invariance, Conservation Laws, and Reductions
}

\author{
P. Masemola and A. H. Kara \\ School of Mathematics, University of the Witwatersrand (Wits), Johannesburg 2050, South Africa
}

Correspondence should be addressed to A. H. Kara; abdul.kara@wits.ac.za

Received 18 March 2014; Accepted 5 August 2014; Published 13 August 2014

Academic Editor: Tao Liu

Copyright (C) 2014 P. Masemola and A. H. Kara. This is an open access article distributed under the Creative Commons Attribution License, which permits unrestricted use, distribution, and reproduction in any medium, provided the original work is properly cited.

An analysis of a PT symmetric coupler with "gain in one waveguide and loss in another" is made; a transformation in the PT system and some assumptions results in a scalar cubic Schrödinger equation. We investigate the relationship between the conservation laws and Lie symmetries and investigate a Lagrangian, corresponding Noether symmetries, conserved vectors, and exact solutions via "double reductions."

\section{Introduction}

Physical systems exhibiting parity-time (PT) symmetry have been the subject of much investigation in recent years and are now extensively considered in diverse areas of physics, namely, quantum field theories, non-Hermitian Anderson models, and complex Lie algebras, just to name a few [110]. We know that even a single PT cell can exhibit unconventional features; it follows that one may wish to investigate what new behaviour and properties can be expected from PT symmetric lattices [11, 12].

In optics, it has recently been discovered that there is a class of optical systems, of which elements consist of gain and loss, that can be interpreted as an optics equivalent of the PT symmetry in quantum mechanics $[11,13]$. The underlying equations describing the effects of pulse dispersion [13] have the following form:

$$
\begin{aligned}
& i U_{t}+U_{x x}+2|U|^{2} U=-V+i \gamma U, \\
& i V_{t}+V_{x x}+2|V|^{2} V=-U-i \gamma V .
\end{aligned}
$$

To analyze the solutions of this equation we make a change of variables

$$
U(x, t)=e^{i(\omega t-\theta)} \alpha(x, t), \quad V(x, t)=e^{i \omega t} \beta(x, t),
$$

where $\theta$ is a constant angle satisfying

$$
\sin \theta=\gamma
$$

and $\omega$ is an arbitrary real parameter. As a result of the transformation, (1) becomes

$$
\begin{aligned}
& i \alpha_{t}+\alpha_{x x}-\omega \alpha+2|\alpha|^{2} \alpha=-\cos \theta \beta+i \gamma(\alpha-\beta), \\
& i \beta_{t}+\beta_{x x}-\omega \beta+2|\beta|^{2} \beta=-\cos \theta \alpha+i \gamma(\alpha-\beta) .
\end{aligned}
$$

The system (4) admits a reduction $\alpha=\beta=q$ to the following scalar cubic Schrödinger equation:

$$
i q_{t}+q_{x x}-a^{2} q+2|q|^{2} q=0
$$

where $q$ is the complex valued dependent variable and $a^{2}=$ $\omega-\cos \theta$.

Equation (5) has a family of stationary soliton solutions; however, we will study the invariance, exact solutions, conservation laws, and double reductions. This will be done by decomposing (5) into real and imaginary parts to obtain the following system of partial differential equations (PDEs); if $q=u+i v$, then we have the following system:

$$
\begin{gathered}
u_{t}+v_{x x}-a^{2} v+2 v\left(u^{2}+v^{2}\right)=0, \\
-v_{t}+u_{x x}-a^{2} u+2 u\left(u^{2}+v^{2}\right)=0 .
\end{gathered}
$$


In light of symmetries, conservation laws, and double reduction to exact solutions, we will briefly consider the system of PDEs in (1) but the bulk of the analysis will centre around (5) via the system (6).

\section{On the Conservation Laws of (1)}

In order to determine conserved densities and fluxes, we resort to the invariance and multiplier approach based on the well-known result that the Euler-Lagrange operator annihilates a total divergence (see [14]). Firstly, if $\left(T^{t}, T^{x}\right)$ is a conserved vector corresponding to a conservation law, then

$$
D_{t} T^{t}+D_{x} T^{x}=0
$$

along the solutions of the differential equation $(G(x, t, q$, $\left.q_{(i)}, \ldots\right)=0$, say).

If the system (1) is split into real and imaginary parts with $U=\mu+i \nu$ and $V=\epsilon+i \delta$ and replacing $\gamma$ with "general" parameters, we get

$$
\begin{aligned}
& \mu_{t}+v_{x x}+2\left(\mu^{2}+v^{2}\right) \nu+\delta-g_{1} \mu=0, \\
& -v_{t}+\mu_{x x}+2\left(\mu^{2}+v^{2}\right) \mu+\epsilon+g_{2} \nu=0, \\
& \epsilon_{t}+\delta_{x x}+2\left(\epsilon^{2}+\delta^{2}\right) \delta+\nu+g_{3} \epsilon=0, \\
& \delta_{t}+\epsilon_{x x}+2\left(\epsilon^{2}+\delta^{2}\right) \epsilon+\mu-g_{4} \delta=0 .
\end{aligned}
$$

It turns out that the system (8) only admits nontrivial conservation laws (two) for $g_{2}=-g_{1}$ and $g_{4}=-g_{3}$ corresponding to multipliers (see below) $Q=\left(v_{t}, \mu_{t}, \delta_{t}, \epsilon_{t}\right)$ and $Q=\left(\nu_{x}, \mu_{x}, \delta_{x}, \epsilon_{x}\right)$ which in turn correspond to time and space translations, respectively. In this case, (8) becomes

$$
\begin{aligned}
& \mu_{t}+\nu_{x x}+2\left(\mu^{2}+v^{2}\right) \nu+\delta-g_{1} \mu=0, \\
& -v_{t}+\mu_{x x}+2\left(\mu^{2}+v^{2}\right) \mu+\epsilon-g_{2} \nu=0, \\
& \epsilon_{t}+\delta_{x x}+2\left(\epsilon^{2}+\delta^{2}\right) \delta+\nu+g_{3} \epsilon=0, \\
& \delta_{t}+\epsilon_{x x}+2\left(\epsilon^{2}+\delta^{2}\right) \epsilon+\mu+g_{3} \delta=0 .
\end{aligned}
$$

Thus, (1) has no nontrivial conservation laws even though the system is invariant under time and space translations.

We thus do a detailed study of the special case given in (6) instead.

\section{Symmetries, Reductions, and Conservation Laws of (6)}

The Lie symmetry approach on differential equations is well known; for details, see, for example, $[15,16]$. In this section, we list a summary of these and explore the notion of a "double reduction" in order to obtain symmetry invariant (exact) solutions.
3.1. Symmetries and Reductions. A one parameter Lie group of transformations that leave invariant (6) will be written as a vector field

$$
\begin{aligned}
X= & \tau(t, x, u, v) \partial_{t}+\xi(t, x, u, v) \partial_{x} \\
& +\eta^{1}(t, x, u, v) \partial_{u}+\eta^{2}(t, x, u, v) \partial_{v} .
\end{aligned}
$$

This would be a generator of point symmetries of the system. We get the algebra generated by

$$
\begin{gathered}
X_{1}=\partial_{t} \\
X_{2}=\partial_{x} \\
X_{3}=u \partial_{v}-v \partial_{u} \\
X_{4}=2 t \partial_{x}+u x \partial_{v}-v x \partial_{u} \\
X_{5}=\left(-2 a^{2} t u-v\right) \partial_{v}+2 t \partial_{t}+\left(-u+2 a^{2} t v\right) \partial_{u}+x \partial_{x} .
\end{gathered}
$$

3.2. Conservation Laws. In order to determine conserved densities and fluxes, we resort to the invariance and multiplier approach based on the well-known result that the EulerLagrange operator annihilates a total divergence (see [14]). Firstly, if $\left(T^{t}, T^{x}\right)$ is a conserved vector corresponding to a conservation law, then

$$
D_{t} T^{t}+D_{x} T^{x}=0
$$

along the solutions of the differential equation $(G(x, t, q$, $\left.q_{(i)}, \ldots\right)=0$, say)

3.2.1. The Mutiplier Approach. If there exists a nontrivial differential function $Q$, called a "multiplier," such that

$$
E_{q}\left[Q G\left(x, t, q, q_{(i)}, \ldots\right)\right]=0
$$

then $\mathrm{Q}\left(G\left(x, t, q, q_{(i)}, \ldots\right)\right)$ is a total divergence; that is,

$$
Q\left(G\left(x, t, q, q_{(i)}, \ldots\right)\right)=D_{t} T^{t}+D_{x} T^{x}
$$

for some (conserved) vector $\left(T^{t}, T^{x}\right)$ and $E_{q}$ is the respective Euler-Lagrange operator. Thus, knowledge of each multiplier $Q$ leads to a conserved vector determined by, inter alia, a homotopy operator. See details and references in [14, 17].

For a system $G^{1}\left(x, t, u, v, u_{(i)}, v_{(i)}, \ldots\right)=0$ and $G^{2}(x, t, u$, $\left.v, u_{(i)}, v_{(i)}, \ldots\right)=0, Q=\left(Q^{1}, Q^{2}\right)$, say, we get

$$
\begin{gathered}
Q^{1}\left(G^{1}\left(x, t, q, u_{(i)}, v_{(i)}, \ldots\right)\right) \\
+Q^{2}\left(G^{2}\left(x, t, q, u_{(i)}, v_{(i)}, \ldots\right)\right)=D_{t} T^{t}+D_{x} T^{x}, \\
E_{(u, v)}\left[D_{t} T^{t}+D_{x} T^{x}\right]=0 .
\end{gathered}
$$

In each case, $T^{t}$ is the conserved density. 
The lengthy calculations for the system (6) lead to the following multipliers and corresponding conserved vectors.

(i) Consider $\left(Q^{1}, Q^{2}\right)=\left(v_{t}, u_{t}\right)$,

$$
\begin{aligned}
T^{x} & =\frac{1}{2}\left(u_{t} u_{x}+v_{t} v_{x}-u u_{x t}-v v_{x t}\right), \\
T^{t} & =\frac{1}{2}\left(u^{4}-u^{2}\left(a^{2}-2 v^{2}\right)+u u_{x x}+v\left(-a^{2} v+v^{3}+v_{x x}\right)\right) .
\end{aligned}
$$

The conserved density for the scalar equation is

$$
\Phi^{t}=\frac{1}{2}|q|^{4}+\frac{1}{2}(h-1)|q|^{2}+q \bar{q}_{x x}+\bar{q} q_{x x} .
$$

(ii) Consider $\left(Q^{1}, Q^{2}\right)=\left(v_{x}, u_{x}\right)$. Consider

$$
\begin{gathered}
T^{x}=\frac{1}{2}\left(u^{4}-a^{2} v^{2}+v^{4}-u^{2}\left(a^{2}-2 v^{2}\right)\right. \\
\left.+v u_{t}-u v_{t}+u_{x}^{2}+v_{x}^{2}\right), \\
T^{t}=\frac{1}{2}\left(-v u_{x}+u v_{x}\right) .
\end{gathered}
$$

The conserved density for the scalar equation is

$$
\Phi^{t}=-\frac{i}{4}\left(\bar{q} q_{x}-q \bar{q}_{x}\right) .
$$

(iii) Consider $\left(Q^{1}, Q^{2}\right)=(u,-v)$,

$$
\begin{gathered}
T^{x}-v u_{x}+u v_{x}, \\
T^{t}=\frac{1}{2}\left(u^{2}+v^{2}\right) .
\end{gathered}
$$

The conserved density for the scalar equation is

$$
\Phi^{t}=-\frac{1}{2}|q|^{2}
$$

(iv) Consider $\left(Q^{1}, Q^{2}\right)=\left(-(1 / 2) x u+t v_{x},(1 / 2) x v+t u_{x}\right)$,

$$
\begin{aligned}
& T^{x}=\frac{1}{2}\left(t u^{4}-a^{2} t v^{2}+t v^{4}-t u^{2}\left(a^{2}-2 v^{2}\right)\right. \\
& \left.+v\left(t u_{t}+x u_{x}\right)-u\left(t v_{t}+x v_{x}\right)+t\left(u_{x}^{2}+v_{x}^{2}\right)\right), \\
& T^{t}=\frac{1}{4}\left(-x u^{2}-v\left(x v+2 t u_{x}\right)+2 t u v_{x}\right) .
\end{aligned}
$$

The conserved density for the scalar equation is

$$
\Phi^{t}=-\frac{1}{4} x|q|^{2}-i t\left(\bar{q} q_{x}-q \bar{q}_{x}\right) .
$$

3.2.2. A Lagrangian Formulation. The system (6) admits a Lagrangian

$$
\begin{aligned}
L= & -\frac{1}{2} u_{x}^{2}-\frac{1}{2} v_{x}^{2}+\frac{1}{2} u_{t} v-\frac{1}{2} v_{t} u \\
& -\frac{1}{2} a^{2}\left(u^{2}+v^{2}\right)+\frac{1}{2} u^{4}+\frac{1}{2} v^{4}+u^{2} v^{2}
\end{aligned}
$$

so that the corresponding Lagrangian for the Schrödinger equation (5) is $\mathscr{L}=-(1 / 2)\left|q_{x}\right|^{2}-(1 / 2) a^{2}|q|^{2}+(1 / 2)|q|^{4}+$ $(i / 4)\left(\bar{q} q_{t}-q \bar{q}_{t}\right)$. The Noether symmetries, that is, the one parameter Lie groups of transformations, that leave invariant the functional $\iint L d x d t$ with zero gauges are the translations

$$
X_{1}=\partial_{t}, \quad X_{2}=\partial_{x}
$$

with corresponding conserved vectors

$$
\begin{aligned}
T^{x}= & v_{x} v_{t}+u_{x} u_{t}, \\
T^{t}= & -\frac{1}{2} u_{x}^{2}-\frac{1}{2} v_{x}{ }^{2}-\frac{1}{2} a^{2} u^{2}-\frac{1}{2} a^{2} v^{2} \\
& +\frac{1}{2} u^{4}+\frac{1}{2} v^{4}+u^{2} v^{2},
\end{aligned}
$$

with density of (5) given by

$$
\Phi^{t}=-\frac{1}{2}\left|q_{x}\right|^{2}-\frac{1}{2} a^{2}|q|^{2}+\frac{1}{2}|q|^{4}
$$

and

$$
\begin{aligned}
T^{x}= & \frac{1}{2} u_{x}{ }^{2}+\frac{1}{2} v_{x}{ }^{2}+\frac{1}{2} v u_{t}-\frac{1}{2} u v_{t}-\frac{1}{2} a^{2} u^{2} \\
& -\frac{1}{2} a^{2} v^{2}+\frac{1}{2} u^{4}+\frac{1}{2} v^{4}+u^{2} v^{2} \\
T^{t}= & \frac{1}{2}\left(v_{x} u-u_{x} v\right)
\end{aligned}
$$

with density of (5) given by

$$
\Phi^{t}=-\frac{i}{4}\left(\bar{q} q_{x}-q \bar{q}_{x}\right)
$$

respectively.

3.3. Double Reduction. To demonstrate how one uses symmetries and conservation laws to double reduce PDEs, we recall some definitions and theorems.

Definition 1 (see [18]). A Lie-Bäcklund symmetry generator $X$ of the form (1) is associated with a conserved vector $T$ of the system (6) if $X$ and $T$ satisfy the relations

$$
X\left(T^{i}\right)+T^{i} D_{k}\left(\xi^{k}\right)-T^{k} D_{k}\left(\xi^{i}\right)=0, \quad i=1, \ldots, n .
$$

Theorem 2 (see [19]). Suppose that $X$ is any Lie-Bäcklund symmetry of (6) and $T^{i}, i=1, \ldots, n$, are the components of the conserved vector of (6). Then

$$
T^{* i}=\left[T^{i}, X\right]=X\left(T^{i}\right)+T^{i} D_{j} \xi^{j}-T^{j} D_{j} \xi^{i}, \quad i=1, \ldots, n,
$$

constitute the components of a conserved vector of (6); that is, $\left.D_{i} T^{* i}\right|_{(6)}=0$. 
Theorem 3 (see [20]). Suppose that $D_{i} T^{i}=0$ is a conservation law of the partial differential equation system (6). Then under a contact transformation, there exist functions $\widetilde{T}^{i}$ such that $J D_{i} T^{i}=\widetilde{D}_{i} \widetilde{T}^{i}$, where $\widetilde{T}^{i}$ are given as

$$
\begin{gathered}
\left(\begin{array}{c}
\widetilde{T}^{1} \\
\widetilde{T}^{2} \\
\vdots \\
\widetilde{T}^{n}
\end{array}\right)=J\left(A^{-1}\right)^{T}\left(\begin{array}{c}
T^{1} \\
T^{2} \\
\vdots \\
T^{n}
\end{array}\right), \\
J\left(\begin{array}{c}
T^{1} \\
T^{2} \\
\vdots \\
T^{n}
\end{array}\right)=A^{T}\left(\begin{array}{c}
\widetilde{T}^{1} \\
\widetilde{T}^{2} \\
\vdots \\
\widetilde{T}^{n}
\end{array}\right)
\end{gathered}
$$

in which

$$
\begin{aligned}
A & =\left(\begin{array}{cccc}
\widetilde{D}_{1} x_{1} & \widetilde{D}_{1} x_{2} & \cdots & \widetilde{D}_{1} x_{n} \\
\widetilde{D}_{2} x_{1} & \widetilde{D}_{2} x_{2} & \cdots & \widetilde{D}_{2} x_{n} \\
\vdots & \vdots & \vdots & \vdots \\
\widetilde{D}_{n} x_{1} & \widetilde{D}_{n} x_{2} & \cdots & \widetilde{D}_{n} x_{n}
\end{array}\right), \\
A^{-1} & =\left(\begin{array}{cccc}
D_{1} \widetilde{x}_{1} & D_{1} \widetilde{x}_{2} & \cdots & D_{1} \widetilde{x}_{n} \\
D_{2} \widetilde{x}_{1} & D_{2} \widetilde{x}_{2} & \cdots & D_{2} \widetilde{x}_{n} \\
\vdots & \vdots & \vdots & \vdots \\
D_{n} \widetilde{x}_{1} & D_{n} \widetilde{x}_{2} & \cdots & D_{n} \widetilde{x}_{n}
\end{array}\right)
\end{aligned}
$$

and $J=\operatorname{det}(A)$.

Theorem 4 (fundamental theorem on double reduction [20]). Suppose that $D_{i} T^{i}=0$ is a conservation law of the partial differential equation system (6). Then under a similarity transformation of a symmetry $X$ of the form (1) for the partial differential equation, there exist functions $\widetilde{T}^{i}$ such that $X$ is still a symmetry for the partial differential equation $\widetilde{D}_{i} \widetilde{T}^{i}=0$ and

$$
\left(\begin{array}{c}
X \widetilde{T}^{1} \\
X \widetilde{T}^{2} \\
\vdots \\
X \widetilde{T}^{n}
\end{array}\right)=J\left(A^{-1}\right)^{T}\left(\begin{array}{c}
{\left[T^{1}, X\right]} \\
{\left[T^{2}, X\right]} \\
\vdots \\
{\left[T^{n}, X\right]}
\end{array}\right),
$$

where

$$
\begin{gathered}
A=\left(\begin{array}{cccc}
\widetilde{D}_{1} x_{1} & \widetilde{D}_{1} x_{2} & \cdots & \widetilde{D}_{1} x_{n} \\
\widetilde{D}_{2} x_{1} & \widetilde{D}_{2} x_{2} & \cdots & \widetilde{D}_{2} x_{n} \\
\vdots & \vdots & \vdots & \vdots \\
\widetilde{D}_{n} x_{1} & \widetilde{D}_{n} x_{2} & \cdots & \widetilde{D}_{n} x_{n}
\end{array}\right), \\
A^{-1}=\left(\begin{array}{ccccc}
D_{1} \widetilde{x}_{1} & D_{1} \widetilde{x}_{2} & \cdots & D_{1} \widetilde{x}_{n} \\
D_{2} \widetilde{x}_{1} & D_{2} \widetilde{x}_{2} & \cdots & D_{2} \widetilde{x}_{n} \\
\vdots & \vdots & \vdots & \vdots \\
D_{n} \widetilde{x}_{1} & D_{n} \widetilde{x}_{2} & \cdots & D_{n} \widetilde{x}_{n}
\end{array}\right)
\end{gathered}
$$

and $J=\operatorname{det}(A)$.
Our original system is equivalent to

$$
\text { sys }_{1}=\left\{\begin{array}{l}
q_{1}{ }^{1} G^{1}+q_{1}{ }^{2} G^{2}=0 \\
q_{1}{ }^{1} G^{1}-q_{1}{ }^{2} G^{2}=0 .
\end{array}\right.
$$

The system (36) can be rewritten as

$$
\begin{aligned}
& D_{t} T_{1}^{t}+D_{x} T_{1}^{x}=0, \\
& q_{1}{ }^{1} G^{1}-q_{1}{ }^{2} G^{2}=0 .
\end{aligned}
$$

3.3.1. A Double Reduction of (6) by $\left\langle X_{2}, X_{3}\right\rangle$. We first show that $X_{2}$ and $X_{3}$ are associated with $T_{1}=\left(T_{2}^{t}, T_{2}^{x}\right)$ using the following version of (6) for $i=1,2$ :

$$
\begin{aligned}
T^{*}= & X\left(\begin{array}{c}
T^{t} \\
T^{x}
\end{array}\right)-\left(\begin{array}{cc}
D_{t} \xi^{t} & D_{x} \xi^{t} \\
D_{t} \xi^{x} & D_{x} \xi^{x}
\end{array}\right)\left(\begin{array}{c}
T^{t} \\
T^{x}
\end{array}\right) \\
& +\left(D_{t} \xi^{t}+D_{x} \xi^{x}\right)\left(\begin{array}{c}
T^{t} \\
T^{x}
\end{array}\right) .
\end{aligned}
$$

We obtain

$$
\begin{aligned}
& \left(\begin{array}{l}
T_{2}^{* t} \\
T_{2}^{* x}
\end{array}\right)=X_{3}^{[1]}\left(\begin{array}{l}
T_{2}^{t} \\
T_{2}^{x}
\end{array}\right)-\left(\begin{array}{ll}
0 & 0 \\
0 & 0
\end{array}\right)\left(\begin{array}{c}
T_{2}^{t} \\
T_{2}^{x}
\end{array}\right)+(0)\left(\begin{array}{l}
T_{2}^{t} \\
T_{2}^{x}
\end{array}\right) \\
& =\left(\begin{array}{c}
\left(-v v_{x}-u u_{x}+v v_{x}+u u_{x}\right) \\
\left.\frac{1}{2}\left(u_{t} u_{x}+v_{t} v_{x}-u u_{x t}-v v_{x t}\right)\right)
\end{array}\right) \\
& =\left(\begin{array}{l}
0 \\
0
\end{array}\right) \text {, } \\
& \left(\begin{array}{c}
T_{2}^{* t} \\
T_{2}^{* *}
\end{array}\right)=X_{2}^{[1]}\left(\begin{array}{c}
T_{2}^{t} \\
T_{2}^{x}
\end{array}\right)-\left(\begin{array}{ll}
0 & 0 \\
0 & 0
\end{array}\right)\left(\begin{array}{c}
T_{2}^{t} \\
T_{2}^{x}
\end{array}\right)+(0)\left(\begin{array}{c}
T_{2}^{t} \\
T_{2}^{x}
\end{array}\right) \\
& =\left(\begin{array}{c}
\frac{\partial}{\partial x}\left[\frac{1}{2}\left(u v_{x}-v u_{x}\right)\right] \\
\frac{\partial}{\partial x}\left[\frac{1}{2}\left(u_{t} u_{x}+v_{t} v_{x}-u u_{x t}-v v_{x t}\right)\right]
\end{array}\right) \\
& =\left(\begin{array}{l}
0 \\
0
\end{array}\right) \text {, }
\end{aligned}
$$

where

$$
\begin{gathered}
X_{3}^{[1]}=-v \frac{\partial}{\partial u}+u \frac{\partial}{\partial v}-v_{t} \frac{\partial}{\partial u_{t}}-v_{x} \frac{\partial}{\partial u_{x}}+u_{t} \frac{\partial}{\partial v_{t}}+u_{x} \frac{\partial}{\partial v_{x}}, \\
X_{2}^{[1]}=\frac{\partial}{\partial x} .
\end{gathered}
$$

Thus, $X_{2}$ and $X_{3}$ are both associated with $T_{2}$.

We can get a reduced conserved form for the first equation of the first system, sys ${ }_{1}$ from (36), since $X_{2}$ and $X_{3}$ are both associated symmetries with $T_{2}$.

We consider a linear combination of $X_{2}$ and $X_{3}$, that is, of the form $X=X_{2}+c X_{3}$, and transform this generator to its canonical form $Y=\partial / \partial s$, where we assume that this generator is of the form $Y=0(\partial / \partial r)+\partial / \partial s+0(\partial / \partial w)+$ $0(\partial / \partial p)$. 
From $X(r)=0, X(s)=1, X(w)=0$, and $X(p)=0$, we obtain

$$
\frac{d t}{0}=\frac{d x}{1}=\frac{d u}{-c v}=\frac{d v}{c u}=\frac{d r}{0}=\frac{d s}{1}=\frac{d w}{0}=\frac{d p}{0} .
$$

We solve (41) using the method of invariance, where the results are summarized as follows:

$$
\begin{aligned}
& b_{1}=t, \quad b_{2}=u^{2}+v^{2}, \quad b_{3}=\arctan \left(\frac{v}{u}\right)-c x, \\
& b_{4}=r, \quad b_{5}=s-x, \quad b_{6}=w, \quad b_{7}=p,
\end{aligned}
$$

where $b_{4}, b_{5}, b_{6}$, and $b_{7}$ are arbitrary functions all dependent on $b_{1}, b_{2}$, and $b_{3}$.

By choosing $b_{4}=b_{1}, b_{5}=0, b_{6}=\sqrt{b_{2}}$, and $b_{7}=b_{3}$, we obtain the canonical coordinates

$$
\begin{gathered}
r=t, \quad s=x, \quad w=\sqrt{u^{2}+v^{2},} \\
p=\arctan \left(\frac{v}{u}\right)-c s,
\end{gathered}
$$

where $w=w(r)$ and $p=p(r)$, since $Y=\partial / \partial s$.

From (32), we compute $A$ and $\left(A^{-1}\right)^{T}$ :

$$
\begin{gathered}
A=\left(\begin{array}{ll}
D_{r} t & D_{r} x \\
D_{s} t & D_{s} x
\end{array}\right)=\left(\begin{array}{ll}
1 & 0 \\
0 & 1
\end{array}\right), \\
A^{-1}=\left(\begin{array}{ll}
D_{t} r & D_{t} s \\
D_{x} r & D_{x} s
\end{array}\right)=\left(\begin{array}{ll}
1 & 0 \\
0 & 1
\end{array}\right)=\left(A^{-1}\right)^{T},
\end{gathered}
$$

where $J=\operatorname{det}(A)=1$.

From (43), the inverse canonical coordinates are given by

$$
\begin{aligned}
t=r, \quad x & =s, \quad u=w(r) \cos (p(r)+c s), \\
v & =w(r) \sin (p(r)+c s) .
\end{aligned}
$$

We compute the first- and second-order partial derivatives of $u$ and $v$ from (45):

$$
\begin{aligned}
u_{t}= & \left(\frac{d}{d r} w(r)\right) \cos (p(r)+c s) \\
& -w(r)\left(\frac{d}{d r} p(r)\right) \sin (p(r)+c s), \\
u_{x}= & -c w(r) \sin (p(r)+c s), \\
v_{t}= & \left(\frac{d}{d r} w(r)\right) \sin (p(r)+c s) \\
& +w(r)\left(\frac{d}{d r} p(r)\right) \cos (p(r)+c s), \\
v_{x}= & c w(r) \cos (p(r)+c s), \\
u_{x x}= & -c^{2} w(r) \cos (p(r)+c s), \\
v_{x x}= & -c^{2} w(r) \sin (p(r)+c s) .
\end{aligned}
$$

We now apply the formula from (33) with $i=1,2$ to obtain the reduced conserved form

$$
\left(\begin{array}{c}
T_{2}^{r} \\
T_{2}^{s}
\end{array}\right)=J\left(A^{-1}\right)^{T}\left(\begin{array}{c}
T_{2}^{t} \\
T_{2}^{x}
\end{array}\right) .
$$

By substituting (45) and (46) into (47), we obtain

$$
\begin{aligned}
& \left(\begin{array}{l}
T_{2}^{r} \\
T_{2}^{s}
\end{array}\right) \\
& =\left(\begin{array}{c}
\frac{1}{2} c w(r)^{2} \\
\frac{1}{2}\left[w(r)^{4}+a^{2} w(r)^{2}-\left(\frac{d}{d r} p(r)\right) w(r)^{2}+c^{2} w(r)^{2}\right]
\end{array}\right),
\end{aligned}
$$

where the reduced conserved form is also given by

$$
D_{r} T_{2}^{r}=0 .
$$

The second step of double reduction can be given as

$$
c w(r)^{2}=k
$$

or equivalently

$$
w(r)^{2}=k,
$$

where $k$ is a constant.

Differentiating (50) implicitly with respect to $r$ results in

$$
\frac{d}{d r} w(r)=0 .
$$

The second equation of sys $s_{1}$ from (36) is given by

$$
\begin{aligned}
v_{x} & {\left[u_{t}+v_{x x}-a^{2} v+2\left(u^{2}+v^{2}\right) v\right] } \\
& -u_{x}\left[-v_{t}+u_{x x}-a^{2} u+2\left(u^{2}+v^{2}\right) u\right]=0 .
\end{aligned}
$$

After transforming (53) using (45) and (46), we obtain

$$
\begin{gathered}
-2 c w(r)^{2} \frac{d}{d r} p(r)[\cos (p(r)+c s) \sin (p(r)+c s)], \\
+4 w(r)^{4}[\cos (p(r)+c s) \sin (p(r)+c s)]=0 .
\end{gathered}
$$

We now substitute (50) and (52) into (54) and simplify.

This results in the ordinary differential equation (ODE)

$$
\frac{d}{d r} p(r)=\frac{2 w(r)^{2}}{c} .
$$

If we substitute (51) into (55) and then integrate both sides with respect to $r$, we obtain

$$
p(r)=\frac{2 k r}{c}+m,
$$

where $m$ is an integration constant.

Using (45), we obtain the final solution to our original system (6) as

$$
\begin{aligned}
& u(t, x)=\sqrt{k} \cos \left(\frac{2 k t}{c}+m+c x\right), \\
& v(t, x)=\sqrt{k} \sin \left(\frac{2 k t}{c}+m+c x\right) .
\end{aligned}
$$

Thus, $q=\sqrt{k} e^{i(2 k t / c+m+c x)}$. 
3.3.2. Case 2: A Reduction of (6) by $X_{4}$ on $T_{3}$. We now show that $X_{4}$ is associated with $T_{3}=\left(T_{3}^{t}, T_{3}^{x}\right)$ using the formula (38).

We obtain

$$
\begin{aligned}
& \left(\begin{array}{c}
T_{3}^{* t} \\
T_{3}^{* x}
\end{array}\right) \\
& =X_{4}^{[1]}\left(\begin{array}{c}
T_{3}^{t} \\
T_{3}^{x}
\end{array}\right)-\left(\begin{array}{ll}
0 & 0 \\
2 & 0
\end{array}\right)\left(\begin{array}{c}
T_{3}^{t} \\
T_{3}^{x}
\end{array}\right)+(0)\left(\begin{array}{c}
T_{3}^{t} \\
T_{3}^{x}
\end{array}\right) \\
& =\left(\begin{array}{c}
\frac{1}{2}(-2 x u v+2 x u v) \\
\left(-x v v_{x}-x u u_{x}+x v v_{x}+v^{2}+x u u_{x}+u^{2}-u^{2}-v^{2}\right)
\end{array}\right) \\
& =\left(\begin{array}{l}
0 \\
0
\end{array}\right),
\end{aligned}
$$

where

$$
\begin{aligned}
X_{4}^{[1]}= & 2 t \frac{\partial}{\partial x}-x v \frac{\partial}{\partial u}+x u \frac{\partial}{\partial v}-\left(x v_{t}+2 u_{x}\right) \frac{\partial}{\partial u_{t}} \\
& -\left(x v_{x}+v\right) \frac{\partial}{\partial u_{x}}+\left(x u_{t}-2 v_{x}\right) \frac{\partial}{\partial v_{t}}+\left(x u_{x}+u\right) \frac{\partial}{\partial v_{x}} .
\end{aligned}
$$

Thus, $X_{4}$ is associated with $T_{3}$.

We again transform the generator $X_{4}$ to its canonical form $Y=\partial / \partial s$.

From $X(r)=0, X(s)=1, X(w)=0$, and $X(p)=0$, we obtain

$$
\frac{d t}{0}=\frac{d x}{2 t}=\frac{d u}{-x v}=\frac{d v}{x u}=\frac{d r}{0}=\frac{d s}{1}=\frac{d w}{0}=\frac{d p}{0} .
$$

The results from solving (60) are summarized as follows:

$$
\begin{aligned}
& b_{1}=t, \quad b_{2}=u^{2}+v^{2}, \quad b_{3}=\arctan \left(\frac{v}{u}\right)-\frac{x^{2}}{4 t}, \\
& b_{4}=r, \quad b_{5}=s-\frac{x}{2 t}, \quad b_{6}=w, \quad b_{7}=p,
\end{aligned}
$$

where $b_{4}, b_{5}, b_{6}$, and $b_{7}$ are arbitrary functions all dependent on $b_{1}, b_{2}$, and $b_{3}$.

By choosing $b_{4}=b_{1}, b_{5}=0, b_{6}=\sqrt{b_{2}}$, and $b_{7}=b_{3}$, we obtain the canonical coordinates

$$
\begin{gathered}
r=t, \quad s=\frac{x}{2 t}, \quad w=\sqrt{u^{2}+v^{2}}, \\
p=\arctan \left(\frac{v}{u}\right)-\frac{x^{2}}{4 t} .
\end{gathered}
$$

From (32), we compute $A$ and $\left(A^{-1}\right)^{T}$ :

$$
\begin{gathered}
A=\left(\begin{array}{ll}
D_{r} t & D_{r} x \\
D_{s} t & D_{s} x
\end{array}\right)=\left(\begin{array}{ll}
1 & 2 s \\
0 & 2 r
\end{array}\right), \\
\left(A^{-1}\right)^{T}=\left(\begin{array}{ll}
D_{t} r & D_{t} s \\
D_{x} r & D_{x} s
\end{array}\right)=\left(\begin{array}{cc}
1 & 0 \\
-\frac{s}{r} & \frac{1}{2 r}
\end{array}\right),
\end{gathered}
$$

where $J=\operatorname{det}(A)=2 r$.
From (62), the inverse canonical coordinates are given by

$$
\begin{gathered}
t=r, \quad x=2 r s, \quad u=w(r) \cos \left(p(r)+r s^{2}\right), \\
v=w(r) \sin \left(p(r)+r s^{2}\right) .
\end{gathered}
$$

We compute the first- and second-order partial derivatives of $u$ and $v$ from (64):

$$
\begin{aligned}
& u_{t}=\left(\frac{d}{d r} w(r)\right) \cos \left(p(r)+r s^{2}\right) \\
& -w(r)\left(\frac{d}{d r} p(r)\right) \sin \left(p(r)+r s^{2}\right) \\
& +s^{2} w(r) \sin \left(p(r)+r s^{2}\right), \\
& u_{x}=-s w(r) \sin \left(p(r)+r s^{2}\right) \text {, } \\
& v_{t}=\left(\frac{d}{d r} w(r)\right) \sin \left(p(r)+r s^{2}\right) \\
& +w(r)\left(\frac{d}{d r} p(r)\right) \cos \left(p(r)+r s^{2}\right) \\
& -s^{2} w(r) \cos \left(p(r)+r s^{2}\right), \\
& v_{x}=s w(r) \cos \left(p(r)+r s^{2}\right) \text {, } \\
& u_{x x}=-\frac{w(r) \sin \left(p(r)+r s^{2}\right)}{2 r} \\
& -s^{2} w(r) \cos \left(p(r)+r s^{2}\right), \\
& v_{x x}=\frac{w(r) \cos \left(p(r)+r s^{2}\right)}{2 r} \\
& -s^{2} w(r) \sin \left(p(r)+r s^{2}\right) .
\end{aligned}
$$

By substituting (64) and (65) into (47), we obtain

$$
\left(\begin{array}{l}
T_{3}^{r} \\
T_{3}^{s}
\end{array}\right)=\left(\begin{array}{c}
r w(r)^{2} \\
0
\end{array}\right),
$$

where the reduced conserved form is also given by

$$
D_{r} T_{3}^{r}=0 \text {. }
$$

The second step of double reduction can be given as

$$
r w(r)^{2}=k
$$

or equivalently

$$
w(r)^{2}=\frac{k}{r}
$$

where $k$ is a constant.

Differentiating (68) implicitly with respect to $r$ results in

$$
2 r w \frac{d}{d r} w(r)+w(r)^{2}=0
$$


or equivalently, after dividing both sides by $2 r$,

$$
w \frac{d}{d r} w(r)+\frac{w(r)^{2}}{2 r}=0 .
$$

The second equation of sys ${ }_{2}$ from (36) is given by

$$
\begin{aligned}
u u_{t} & -v v_{t}+u v_{x x}+v u_{x x} \\
& -2 a^{2} u v+4 u^{3} v+4 v^{3} u=0 .
\end{aligned}
$$

After transforming (72) using (64) and (65) and multiplying both sides by $2 r$, we get the ODE

$$
\frac{d}{d r} p(r)=2 k-a^{2}
$$

If we substitute (69) into (73) and then integrate both sides with respect to $r$,

$$
p(r)=2 k r-r a^{2}+m
$$

Using (64), we obtain the final solution to our original system (6) as

$$
\begin{aligned}
& u(t, x)=\sqrt{\frac{k}{t}} \cos \left(2 k t-t a^{2}+m+\frac{x^{2}}{4 t}\right), \\
& v(t, x)=\sqrt{\frac{k}{t} \sin \left(2 k t-t a^{2}+m+\frac{x^{2}}{4 t}\right),}
\end{aligned}
$$

so that $q=\sqrt{k / t} e^{i\left(2 k t-t a^{2}+m+x^{2} / 4 t\right)}$.

\section{Conclusion}

We have constructed conservation laws for the scalar cubic Schrödinger equation via the invariance and multiplier approach based on the well-known result that the EulerLagrange operator annihilates total divergence. Interestingly enough, the scalar cubic Schrödinger equation admits a Lagrangian resulting in Noether symmetries. Furthermore, two cases of double reduction were successfully performed and exact solutions were calculated and diagrammatically represented.

\section{Conflict of Interests}

The authors declare that there is no conflict of interests regarding the publication of this paper.

\section{References}

[1] K. G. Makris, R. El-Ganainy, Z. H. Musslimani, and D. N. Christodoulides, "Theory of coupled optical PT-symmetric structures," Optics Letters, vol. 32, no. 17, pp. 2632-2634, 2007.

[2] I. V. Barashenkov, E. V. Zemlyanaya, and T. C. van Heerden, "Time-periodic solitons in a damped-driven nonlinear Schrödinger equation," Physical Review E-Statistical, Nonlinear, and Soft Matter Physics, vol. 83, no. 5, Article ID 056609, 2011.
[3] I. V. Barashenkov and E. V. Zemlyanaya, "Soliton complexity in the damped-driven nonlinear Schrödinger equation: stationary to periodic to quasiperiodic complexes," Physical Review E, vol. 83, no. 5, Article ID 056610, 2011.

[4] J. W. Miles, "Parametrically excited solitary waves," Journal of Fluid Mechanics, vol. 148, pp. 451-460, 1984.

[5] E. W. Laedke and K. H. Spatschek, "On localized solutions in nonlinear Faraday resonance," Journal of Fluid Mechanics, vol. 223, pp. 589-601, 1991.

[6] I. V. Barashenkov, N. V. Alexeeva, and E. V. Zemlyanaya, "Two- and three-dimensional oscillons in nonlinear Faraday resonance," Physical Review Letters, vol. 89, no. 10, Article ID 104101, 2002.

[7] M. G. Clerc, S. Coulibaly, and D. Laroze, "Localized states beyond the asymptotic parametrically driven amplitude equation," Physical Review E-Statistical, Nonlinear, and Soft Matter Physics, vol. 77, no. 5, Article ID 056209, 2008.

[8] I. H. Deutsch and I. Abram, "Reduction of quantum noise in soliton propagation by phase-sensitive amplification," Journal of the Optical Society of America B, vol. 11, no. 11, pp. 2303-2313, 1994.

[9] I. V. Barashenkov, M. M. Bogdan, and V. I. Korobov, "Stability diagram for the phase-locked soliton of the parametrically driven-damped nonlinear Shrödinger equation," Europhysics Letters, vol. 15, pp. 113-118, 1991.

[10] M. G. Clerc, S. Coulibaly, and D. Laroze, "Localized states and non-variational Ising-Bloch transition of a parametrically driven easy-plane ferromagnetic wire," Physica D, vol. 239, no. 1-2, pp. 72-86, 2010.

[11] K. G. Makris, R. El-Ganainy, D. N. Christodoulides, and Z. H. Musslimani, "Beam dynamics in PT symmetric optical lattices," Physical Review Letters, vol. 100, no. 10, Article ID 103904, 2008.

[12] C. M. Bender and S. Boettcher, "Real spectra in non-Hermitian Hamiltonians having PT symmetry," Physical Review Letters, vol. 80, no. 24, pp. 5243-5246, 1998.

[13] N. V. Alexeeva, I. V. Barashenkov, A. A. Sukhorukov, and Y. S. Kivshar, "Optical solitons in PT-symmetric nonlinear couplers with gain and loss," Physical Review A-Atomic, Molecular, and Optical Physics, vol. 85, no. 6, Article ID 063837, 2012.

[14] U. Göktas and W. Hereman, "Computation of conservation laws for nonlinear lattices," Physica D, vol. 123, no. 1-4, pp. 425-436, 1998.

[15] G. W. Bluman and S. Kumei, Symmetries and Differential Equations, Springer, New York, NY, USA, 1989.

[16] P. J. Olver, Applications of Lie Groups to Differential Equations, vol. 107 of New York, NY, USA, Springer, 2nd edition, 1993.

[17] A. H. Kara, "A symmetry invariance analysis of the multipliers and conservation laws of the Jaulent-Miodek and some families of systems of KdV type equations," Journal of Nonlinear Mathematical Physics, vol. 16, pp. 149-156, 2009.

[18] A. H. Kara and F. M. Mahomed, "Relationship between symmetries and conservation laws," International Journal of Theoretical Physics, vol. 39, no. 1, pp. 23-40, 2000.

[19] A. H. Kara and F. M. Mahomed, "A basis of conservation laws for partial differential equations," Journal of Nonlinear Mathematical Physics, vol. 9, pp. 60-72, 2002.

[20] A. H. Bokhari, A. Y. Al-Dweik, F. D. Zaman, A. H. Kara, and F. M. Mahomed, "Generalization of the double reduction theory," Nonlinear Analysis: Real World Applications, vol. 11, no. 5, pp. 3763-3769, 2010. 


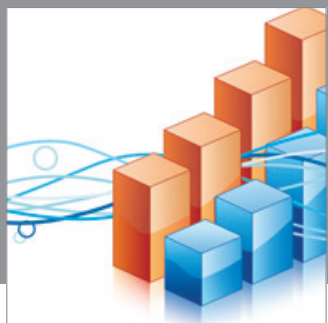

Advances in

Operations Research

mansans

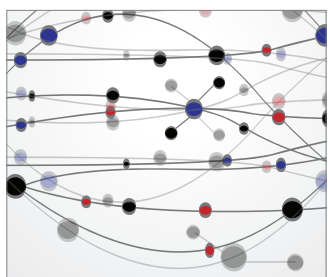

The Scientific World Journal
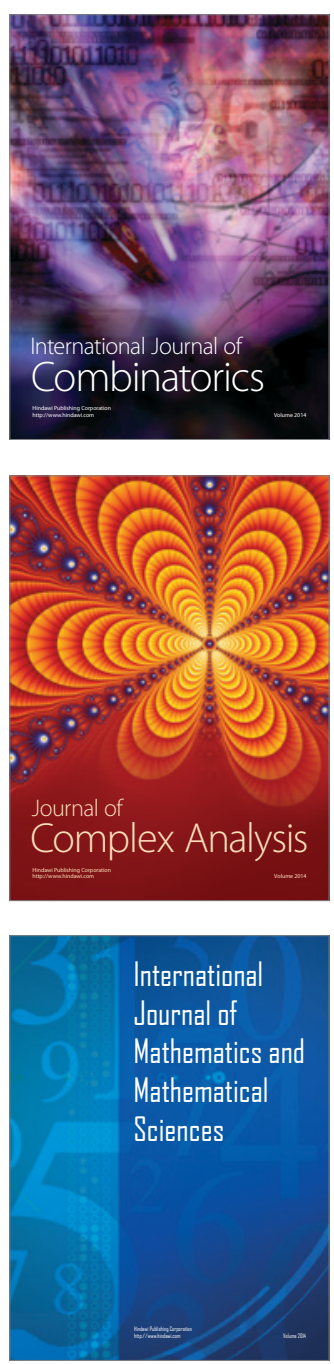
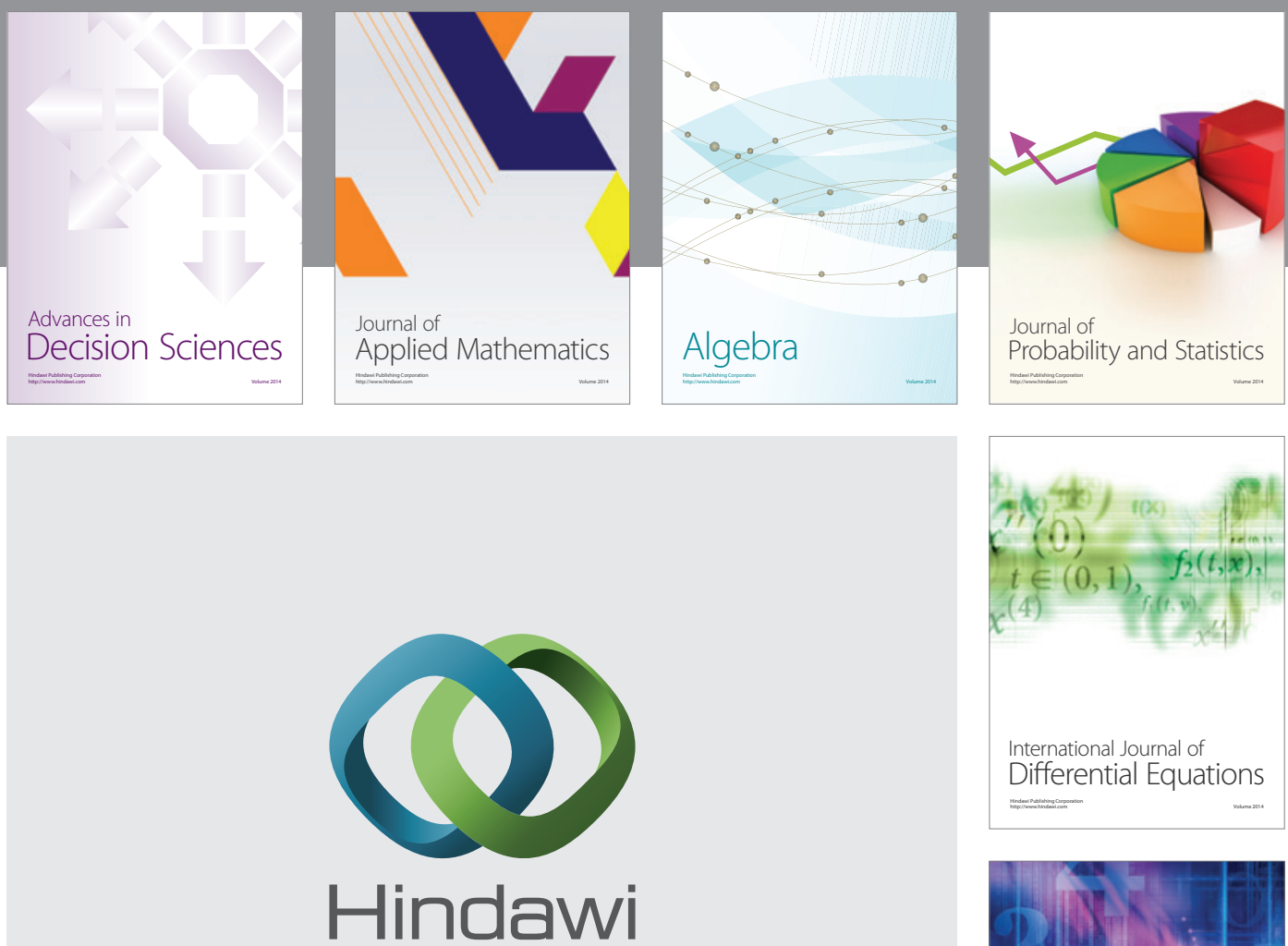

Submit your manuscripts at http://www.hindawi.com
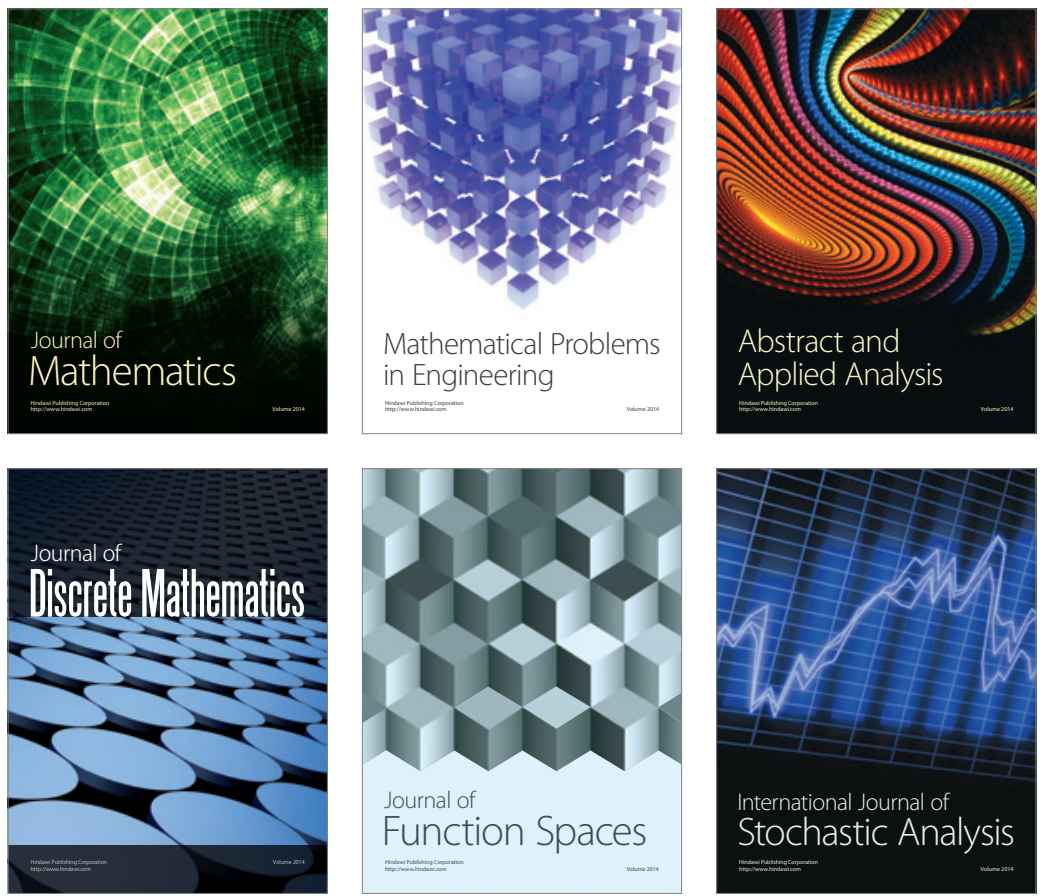

Journal of

Function Spaces

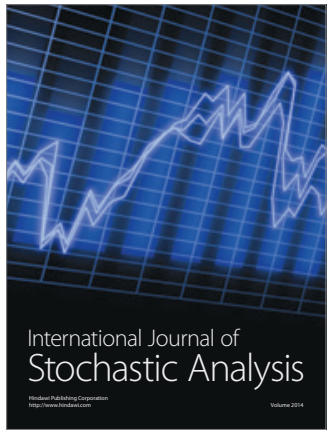

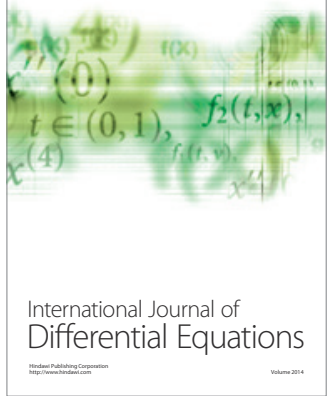
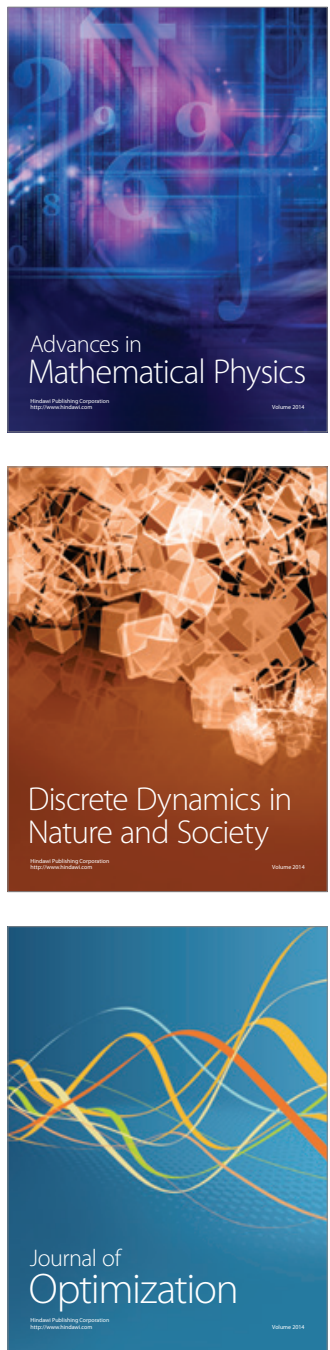